\title{
Coloring Complete and Complete Bipartite Graphs from Random Lists
}

\author{
Carl Johan Casselgren and Roland Haggkvist
}

\section{Linköping University Post Print}

\section{Tweet}

N.B.: When citing this work, cite the original article.

The original publication is available at www.springerlink.com:

Carl Johan Casselgren and Roland Haggkvist, Coloring Complete and Complete Bipartite Graphs from Random Lists, 2016, Graphs and Combinatorics, (32), 2, 533-542.

http://dx.doi.org/10.1007/s00373-015-1587-5

Copyright: Springer Verlag (Germany)

http://www.springerlink.com/?MUD=MP

Postprint available at: Linköping University Electronic Press

http://urn.kb.se/resolve?urn=urn:nbn:se:liu:diva-126248 


\title{
Coloring complete and complete bipartite graphs from random lists
}

\author{
Carl Johan Casselgren* \\ Department of Mathematics \\ Linköping University \\ SE-581 83 Linköping, Sweden
}

\author{
Roland Häggkvist ${ }^{\dagger}$ \\ Department of Mathematics \\ Umeå University \\ SE-901 87 Umeå, Sweden
}

\begin{abstract}
Assign to each vertex $v$ of the complete graph $K_{n}$ on $n$ vertices a list $L(v)$ of colors by choosing each list independently and uniformly at random from all $f(n)$-subsets of a color set $[n]=\{1, \ldots, n\}$, where $f(n)$ is some integer-valued function of $n$. Such a list assignment $L$ is called a random $(f(n),[n])$-list assignment. In this paper, we determine the asymptotic probability (as $n \rightarrow \infty$ ) of the existence of a proper coloring $\varphi$ of $K_{n}$, such that $\varphi(v) \in L(v)$ for every vertex $v$ of $K_{n}$. We show that this property exhibits a sharp threshold at $f(n)=\log n$. Additionally, we consider the corresponding problem for the line graph of a complete bipartite graph $K_{m, n}$ with parts of size $m$ and $n$, respectively. We show that if $m=o(\sqrt{n}), f(n) \geq 2 \log n$, and $L$ is a random $(f(n),[n])$-list assignment for the line graph of $K_{m, n}$, then with probability tending to 1 , as $n \rightarrow \infty$, there is a proper coloring of the line graph of $K_{m, n}$ with colors from the lists.
\end{abstract}

Keywords: list coloring, random list, coloring from random lists, complete graph, complete bipartite graph

\section{Introduction}

Given a graph $G$, assign to each vertex $v$ of $G$ a set $L(v)$ of colors (positive integers). Such an assignment $L$ is called a list assignment for $G$ and the sets $L(v)$ are referred to as lists or color lists. If all lists have equal size $k$, then $L$ is called a $k$-list assignment. We then want to find a proper vertex coloring $\varphi$ of $G$, such that $\varphi(v) \in L(v)$ for all $v \in V(G)$. If such a coloring $\varphi$ exists then $G$ is $L$-colorable and $\varphi$ is called an $L$-coloring. Furthermore, $G$ is called $k$-choosable if it is $L$-colorable for every $k$-list assignment $L$. The least number $k$ such that $G$ is $k$-choosable is called the list-chromatic number of $G$ and is denoted by $\chi_{l}(G)$. We denote by $\chi(G)$ the chromatic number of a graph $G$, i.e. the minimum number $k$ such that there is a proper $k$-coloring of $G$.

This particular variant of vertex coloring is known as list coloring and was introduced by Vizing [11] and independently by Erdős et al. [6]. The problem of list coloring edges of a graph $G$ is

*E-mail address: carl.johan.casselgren@liu.se

${ }^{\dagger}$ E-mail address: roland.haggkvist@math.umu.se 
completely analogous to the vertex coloring case, and can be defined as a (vertex) list coloring problem of the line graph $\mathcal{L}(G)$ of $G$.

In this paper we continue the study of list coloring of graphs from random lists. For an integervalued function $f(n)$ and a set $A$, a random $(f(n), A)$-list assignment for a graph $G$ is a list assignment for $G$ where the list for each vertex is selected independently and uniformly at random from all $f(n)$-subsets of $A$. The following problem was first studied by Krivelevich and Nachmias $[9,10]$ for the case of complete bipartite graphs with parts of equal size $n$ and powers of cycles with bounded maximum degree. An event $A_{n}$ occurs with high probability, abbreviated whp, if $\lim _{n \rightarrow \infty} \mathbb{P}\left[A_{n}\right]=1$.

Problem 1.1. Let $G=G(n)$ be a graph on $n$ vertices, $k$ a fixed positive integer and $\mathcal{C}=\{1, \ldots, \sigma\}$. Suppose that $L$ is a random $(k, \mathcal{C})$-list assignment for $G$. How large should $\sigma=\sigma(n)$ be in order to guarantee that whp $G$ is $L$-colorable?

In [2] the above problem is studied for the case of complete multipartite graphs, and in $[3,4,5]$ some results for graphs with bounded maximum degree (generalizing results from [9]) and complete graphs are obtained. In this note we consider a variation of the above problem as follows:

Problem 1.2. Let $G=G(n)$ be a graph with chromatic number $n$ and $f(n)$ some integer-valued function of $n$. Suppose that $L$ is a random $(f(n),\{1, \ldots, n\})$-list assignment for $G$. How large should $f(n)$ be in order to guarantee that whp $G$ has an $L$-coloring?

In [1] Problem 1.2 is studied for the case of the line graph of a complete bipartite graph $K_{n, n}$ with parts of size $n$. By König's edge coloring theorem $\chi(\mathcal{L}(G))=\Delta(G)$ for any bipartite graph $G$, where $\Delta(G)$ is the maximum degree of $G$. Thus $\chi\left(\mathcal{L}\left(K_{n, n}\right)\right)=n$. In fact, Galvin [7] proved that $\chi_{l}\left(\mathcal{L}\left(K_{n, n}\right)\right)=n$; so if all lists have size $n$ in a list assignment for $\mathcal{L}\left(K_{n, n}\right)$, then we are always guaranteed a proper coloring from the lists. In [1] it is proved that there is a constant $c>0$ such that if $L$ is a random $((1-c) n,\{1, \ldots, n\})$-list assignment ${ }^{1}$ for $\mathcal{L}\left(K_{n, n}\right)$, then whp there is an $L$-coloring of $\mathcal{L}\left(K_{n, n}\right)$. Note that in [1] this result is formulated in the language of arrays and Latin squares.

In this paper, we study Problem 1.2 for the case of complete graphs and line graphs of complete bipartite graphs. Our first result is that for the complete graph $K_{n}$ the property of being colorable from a random $(f(n),\{1, \ldots, n\})$-list assignment has a sharp threshold at $f(n)=\log n$, where $\log$ denotes the natural logarithm.

Theorem 1.3. Let $L$ be a random $(c \log n,\{1, \ldots, n\})$-list assignment for the complete graph $K_{n}$, where $c$ is a constant. If $c>1$, then whp there is an L-coloring of $K_{n}$, and if $c<1$, then whp there is no L-coloring of $K_{n}$.

Since $K_{n} \subseteq \mathcal{L}\left(K_{n, n}\right), \log n$ is a lower bound on the list size $f(n)$ in a random $(f(n),\{1, \ldots, n\})$ list assignment $L$ for $\mathcal{L}\left(K_{n, n}\right)$ such that whp $\mathcal{L}\left(K_{n, n}\right)$ is $L$-colorable. We would like to propose the following conjecture.

Conjecture 1.4. There is a constant $c>1$ such that if $L$ is a random $(c \log n,\{1, \ldots, n\})$-list assignment for the edges of the complete bipartite graph $K_{n, n}$, then whp there is an L-coloring of the edges of $K_{n, n}$.

We are, however, only able to prove the following theorem which shows that a weaker version of the conjecture is true.

\footnotetext{
${ }^{1}$ In order for this to make proper sense, $(1-c) n$ should be replaced by $\lceil(1-c) n\rceil$ or $\lfloor(1-c) n\rfloor$. However, for simplicity of notation, we usually omit floor and ceiling signs.
} 
Theorem 1.5. There is a constant $c>1$, such that if $m=o(\sqrt{n})$ and $L$ is a random $(c \log n,\{1, \ldots, n\})$ list assignment for the edges of the complete bipartite graph $K_{m, n}$, then whp there is an L-coloring of the edges of $K_{m, n}$.

In the remaining part of the paper we prove these two theorems. Throughout the paper, our asymptotic notation and assumptions are standard. In particular, we assume that the parameter $n$ is large enough whenever necessary.

\section{Complete graphs}

In this section we prove Theorem 1.3. Let $L$ be a random $(c \log n,\{1, \ldots, n\})$-list assignment for $K_{n}$, where $c$ is some fixed constant. We will show that if $c>1$, then whp $K_{n}$ has an $L$-coloring, and if $c<1$, then whp $K_{n}$ is not $L$-colorable. To this end, we form a random bipartite graph $B$ by letting $V\left(K_{n}\right)$ and $\{1, \ldots, n\}$ be the partite sets of $B$, and letting $v \in V\left(K_{n}\right)$ and $i \in\{1, \ldots, n\}$ be adjacent if $i \in L(v)$. Clearly, $K_{n}$ is $L$-colorable if and only if there is a perfect matching in $B$. Note that the degree of a vertex in $V\left(K_{n}\right)$ is $c \log n$ in $B$.

We first show that if $c<1$, then whp $B$ contains some isolated vertex, and if $c>1$, then whp $B$ has no isolated vertex. For the second statement, let $X$ be a random variable counting the number of isolated vertices in $B$ and note that

$$
\mathbb{E}[X]=n \frac{\left(\begin{array}{c}
n-1 \\
c \log n
\end{array}\right)^{n}}{\left(\begin{array}{c}
n \\
c \log n
\end{array}\right)^{n}} \sim n \exp (-c \log n)=n^{1-c},
$$

which tends to 0 as $n \rightarrow \infty$ if $c>1$. Thus, by Markov's inequality $B$ whp has no isolated vertex when $c>1$.

To show that whp $B$ has some isolated vertex if $c<1$, we use Chebyshev's inequality in the following form:

$$
\mathbb{P}[Y=0] \leq \frac{\mathbb{E}\left[Y^{2}\right]-\mathbb{E}[Y]^{2}}{\mathbb{E}[Y]^{2}},
$$

valid for all non-negative random variables $Y$. Since $X$ is a sum of $n$ identically distributed indicator random variables, we have

$$
\mathbb{E}\left[X^{2}\right]=\mathbb{E}[X]+n(n-1) \mathbb{E}\left[X_{1} X_{2}\right],
$$

where $X_{1}$ and $X_{2}$ are the indicator random variables for the events that vertex 1 and 2 in $B$ are isolated, respectively. Moreover, since

$$
\mathbb{E}\left[X_{1} X_{2}\right]=\frac{\left(\begin{array}{c}
n-2 \\
c \log n
\end{array}\right)^{n}}{\left(\begin{array}{c}
n \\
c \log n
\end{array}\right)^{n}} \sim n^{-2 c},
$$

we get that

$$
\mathbb{E}\left[X^{2}\right] \leq \mathbb{E}[X]+\frac{n(n-1)}{n^{2 c}} .
$$

By (1), $\mathbb{E}[X] \rightarrow \infty$ if $c<1$, so it now follows from (1)-(3) that $\mathbb{P}[X=0]=o(1)$ when $c<1$. Consequently, if $c<1$, then whp there is no perfect matching in $B$.

We now show that if $c>1$, then whp $B$ has a perfect matching. If $B$ does not have a perfect matching, then there is some set $T$ of vertices in $B$ that violates Hall's condition, i.e. a subset $T$ of $V\left(K_{n}\right)$ or $\{1, \ldots, n\}$ such that $\left|N_{B}(T)\right|<|T|$, where $N_{B}(T)$ is the set of vertices in $B$ that are adjacent to at least one vertex in $T$. If we choose such a minimal set $S$, then 
(i) $\left|N_{B}(S)\right|=|S|-1$,

(ii) $|S| \leq\lceil n / 2\rceil$, and

(iii) each vertex in $N_{B}(S)$ is adjacent to at least two vertices in $S$.

For $s=1,2, \ldots$, let $Z_{s}$ be a random variable counting the number of minimal sets $S \subseteq\{1, \ldots, n\}$ in $B$ of size $s$ that violate Hall's condition, and let $Y_{s}$ be a random variable counting the number of minimal sets $S \subseteq V\left(K_{n}\right)$ in $B$ of size $s$ that violate Hall's condition. We set

$$
Z=\sum_{s=1}^{\lceil n / 2\rceil} Z_{s} \text { and } Y=\sum_{s=1}^{\lceil n / 2\rceil} Y_{s}
$$

and first consider the random variable $Z$.

Since $X=Z_{1}$, we have that $\mathbb{P}\left[Z_{1}>0\right]=o(1)$. If $s=2$, then $S$ consists of two vertices $u, v$ with degree 1 in $B$, which in $B$ are adjacent to the same vertex in $V\left(K_{n}\right)$. Hence,

$$
\begin{aligned}
\mathbb{E}\left[Z_{2}\right] & \leq \frac{n^{3}\left(\begin{array}{c}
n-2 \\
c \log n-2
\end{array}\right)\left(\begin{array}{c}
n-2 \\
c \log n
\end{array}\right)^{n-1}}{\left(\begin{array}{c}
n \\
c \log n
\end{array}\right)^{n}} \\
& =O\left(n \log ^{2} n\right) \exp \left(-2 \frac{n-1}{n} c \log n\right)
\end{aligned}
$$

which tends to 0 as $n \rightarrow \infty$, because $c>1$. Consider now the case when $s \geq 3$. There are $\left(\begin{array}{l}n \\ s\end{array}\right)$ ways of choosing the set $S,\left(\begin{array}{c}n \\ s-1\end{array}\right)$ ways of choosing the set $N_{B}(S)$, and at most $\left(\begin{array}{l}s \\ 2\end{array}\right)^{s-1}$ ways of realizing (iii). Note further that each vertex in $V\left(K_{n}\right) \backslash N_{B}(S)$ cannot be adjacent to a vertex in $S$. We thus have

$$
\begin{aligned}
\sum_{s=3}^{\lceil n / 2\rceil} \mathbb{E}\left[Z_{s}\right] & \leq \sum_{s=3}^{\lceil n / 2\rceil} \frac{\left(\begin{array}{c}
n \\
s
\end{array}\right)\left(\begin{array}{c}
n \\
s-1
\end{array}\right)\left(\begin{array}{c}
s \\
2
\end{array}\right)^{s-1}\left(\begin{array}{c}
n-2 \\
c \log n-2
\end{array}\right)^{s-1}\left(\begin{array}{c}
n-s \\
c \log n
\end{array}\right)^{n-s+1}}{\left(\begin{array}{c}
n \\
c \log n
\end{array}\right)^{n}} \\
& \leq \sum_{s=3}^{\lceil n / 2\rceil}\left(\frac{e n}{s}\right)^{s}\left(\frac{e n}{s-1}\right)^{s-1} s^{2(s-1)}\left(\frac{c^{2} \log ^{2} n}{n(n-1)}\right)^{s-1} \exp \left(-\frac{s}{n}(n-s+1) c \log n\right) \\
& =O(n) \sum_{s=3}^{\lceil n / 2\rceil} e^{2 s}(c \log n)^{2 s} \exp \left(-\frac{s}{n}(n-s+1) c \log n\right) \\
& =O(n) \sum_{s \geq 3}\left(\frac{(c e)^{2} \log ^{2} n}{n^{c / 2}}\right)^{s}=o(1) .
\end{aligned}
$$

Hence, $\mathbb{P}[Z>0]=o(1)$.

Let us now verify that whp there is no set $S \subseteq V\left(K_{n}\right)$ in $B$ that violates Hall's condition. Since all neighbors of a vertex in $S$ are in $N_{B}(S)$, we must have $s \geq c \log n+1$, and there are at most

$$
\left(\begin{array}{l}
n \\
s
\end{array}\right)\left(\begin{array}{c}
n \\
s-1
\end{array}\right)\left(\begin{array}{c}
s-1 \\
c \log n
\end{array}\right)^{s}
$$

realizations of the subgraph induced by $S \cup N_{B}(S)$ in $B$, if $S \subseteq V\left(K_{n}\right)$. We thus conclude 


$$
\begin{aligned}
\sum_{s=c \log n+1}^{\lceil n / 2\rceil} \mathbb{E}\left[Y_{s}\right] & \leq \sum_{s=c \log n+1}^{\lceil n / 2\rceil} \frac{\left(\begin{array}{c}
n \\
s
\end{array}\right)\left(\begin{array}{c}
n \\
s-1
\end{array}\right)\left(\begin{array}{c}
s-1 \\
c \log n
\end{array}\right)^{s}\left(\begin{array}{c}
n \\
c \log n
\end{array}\right)^{n-s}}{\left(\begin{array}{c}
n \\
c \log n
\end{array}\right)^{n}} \\
& =O(1) \sum_{s=c \log n+1}^{\lceil n / 2\rceil}\left(\frac{e n}{s}\right)^{s}\left(\frac{e n}{s-1}\right)^{s-1}\left(\frac{s-1}{n}\right)^{s c \log n} .
\end{aligned}
$$

We split this sum into the two terms

$$
\Delta_{1}=\sum_{s=c \log n+1}^{n / w_{n}+1}\left(\frac{e n}{s}\right)^{s}\left(\frac{e n}{s-1}\right)^{s-1}\left(\frac{s-1}{n}\right)^{s c \log n}
$$

and

$$
\Delta_{2}=\sum_{s=n / w_{n}+2}^{\lceil n / 2\rceil}\left(\frac{e n}{s}\right)^{s}\left(\frac{e n}{s-1}\right)^{s-1}\left(\frac{s-1}{n}\right)^{s c \log n}
$$

where $w_{n}$ is some function such that $w_{n} \rightarrow \infty$ arbitrarily slowly as $n \rightarrow \infty$. The first sum $\Delta_{1}$ is easily seen to satisfy

$$
\Delta_{1} \leq \sum_{s \geq c \log n+1} \frac{e^{2 s-1} n^{2 s-1}}{(s-1)^{2 s-1} n^{s c \log w_{n}}}=o(1),
$$

provided that $n$ is large enough. As for $\Delta_{2}$, we have

$$
\Delta_{2} \leq \sum_{s \geq n / w_{n}+1} \frac{e^{2 s-1} w_{n}^{2 s-1}}{n^{s c \log 2}}=o(1) .
$$

Hence, we conclude that $\mathbb{P}[Y>0]=o(1)$. In other words, whp there is no subset of $V\left(K_{n}\right)$ in $B$ that does not satisfy Hall's condition. This completes the proof of Theorem 1.3.

Remark 2.1. The result proved above should not be too surprising, once it is recalled that in a random bipartite graph distributed as $\mathcal{G}(n, n, p)$, i.e. with parts of size $n$ and where each edge occurs with probability $p$ independently of all other edges, the property of having a perfect matching has a sharp threshold at $p=\log n / n$ (see e.g. [8]). (We remark that we have not been able to deduce Theorem 1.3 directly from this property of $\mathcal{G}(n, n, p)$.) Additionally, an immediate consequence of the sharp threshold for the model $\mathcal{G}(n, n, p)$ is the following: Consider a list assignment $L$ for $K_{n}$, such that each color in $\{1, \ldots, n\}$ appears in each list with probability $p$, independently of each other. By forming a bipartite graph as in the proof above, we get exactly a random bipartite graph distributed as $\mathcal{G}(n, n, p)$, and thus the property of being colorable from such a random list assignment has a sharp threshold at $p=\log n / n$ for the complete graph $K_{n}$.

\section{Complete bipartite graphs}

In this section we prove Theorem 1.5. We have to show that there is a constant $c>1$ such that if $m=o(\sqrt{n})$ and $L$ is a random $(c \log n,\{1, \ldots, n\})$-list assignment for the edges of $K_{m, n}$, then 
whp there is an $L$-coloring of the edges of $K_{m, n}$. In fact, we will prove that $c \geq 2$ suffices. $^{2}$ Suppose that the partite sets of $K_{m, n}$ are $U=\left\{x_{1}, \ldots, x_{m}\right\}$ and $W=\left\{y_{1}, \ldots, y_{n}\right\}$, and let $L$ be a random $(c \log n,\{1, \ldots, n\})$-list assignment for $K_{m, n}$. Our proof in this section is similar to the proof of Theorem 1.3. We define $m$ random bipartite graphs $B_{1}, \ldots, B_{m}$ as follows: the partite sets of each $B_{i}$ are $V_{1}^{(i)}=\{1, \ldots, n\}$ and $V_{2}^{(i)}=\{1, \ldots, n\}$, and $j k \in E\left(B_{i}\right)$ if $k \in L\left(x_{i} y_{j}\right)$. Set $B=B_{1} \cup \cdots \cup B_{m}$. Note that $B$ is a random bipartite multigraph and that the degree of each vertex in $V_{1}^{(i)}$ is $c \log n$ in $B_{i}$, for each $i=1, \ldots, m$.

For a multigraph $G$, we will say that two matchings $M$ and $M^{\prime}$ in $G$ are non-overlapping if there are no two edges $e, e^{\prime}$ such that $e \in M$ and $e^{\prime} \in M^{\prime}$, and $e$ and $e^{\prime}$ have the same pair of ends in $G$. It is straightforward to verify that there is an $L$-coloring of the edges of $K_{m, n}$ if and only if there are pairwise non-overlapping perfect matchings $M_{1}, \ldots, M_{m}$ in $B$, such that $M_{i} \subseteq E\left(B_{i}\right)$, for each $i=1, \ldots, m$.

Let $A_{1}$ be the event that there is a perfect matching $M_{1} \subseteq E\left(B_{1}\right)$ in $B$ and for each integer $i$ such that $1<i \leq m$, let $A_{i}$ be the event that there are perfect matchings $M_{1}, \ldots, M_{i}$ such that for $j=1, \ldots, i, M_{j} \subseteq E\left(B_{j}\right)$, and $M_{1}, \ldots, M_{i}$ are pairwise non-overlapping. We will prove that $\mathbb{P}\left[A_{m}\right]=1-o(1)$. Since $A_{i+1} \subseteq A_{i}$ if $1 \leq i<m, \mathbb{P}\left[A_{m}\right]=\mathbb{P}\left[A_{1} \cap \cdots \cap A_{m}\right]$, and thus

$$
\mathbb{P}\left[A_{m}\right]=\mathbb{P}\left[A_{1}\right] \mathbb{P}\left[A_{2} \mid A_{1}\right] \ldots \mathbb{P}\left[A_{m} \mid A_{1} \cap \cdots \cap A_{m-1}\right] .
$$

Hence, to prove the theorem it suffices to show that

$$
\mathbb{P}\left[A_{1}\right] \mathbb{P}\left[A_{2} \mid A_{1}\right] \ldots \mathbb{P}\left[A_{m} \mid A_{1} \cap \cdots \cap A_{m-1}\right]=1-o(1),
$$

if $m=o(\sqrt{n})$. Since $\mathcal{L}\left(K_{1, n}\right)=K_{n}$, Theorem 1.3 implies that $\mathbb{P}\left[A_{1}\right]=1-o(1)$, if $c \geq 2$. We will now show that there is a constant $K$ such that if $c \geq 2,1 \leq r<m$, and $m=o(\sqrt{n})$, then

$$
\mathbb{P}\left[A_{r+1} \mid A_{1} \cap \cdots \cap A_{r}\right] \geq 1-\frac{K}{\sqrt{n}} .
$$

Since $m=o(\sqrt{n}),\left(1-\frac{K}{\sqrt{n}}\right)^{m} \rightarrow 1$ as $n \rightarrow \infty$, so this suffices to prove (5).

So suppose that there are $r$ pairwise non-overlapping perfect matchings $M_{1}, \ldots, M_{r}$ in $B$, such that $M_{i} \subseteq E\left(B_{i}\right), i=1, \ldots, r$. For all potential edges of $B_{r+1}$, we say that an edge is unusable if it has the same pair of ends as an edge in $M_{1} \cup \cdots \cup M_{r}$. Otherwise a potential edge of $B_{r+1}$ is usable. We need to show that the probability that there is no perfect matching in $B_{r+1}$ containing only usable edges is at most $\Theta\left(n^{-1 / 2}\right)$, and will proceed similarly as in the proof of Theorem 1.3.

Let $N_{B_{r+1}}^{\prime}(S)$ be the set of neighbors of $S$ via usable edges in $B_{r+1}$, i.e. a vertex $v$ which is adjacent to some vertex in $S$ via a usable edge is in $N_{B_{r+1}}^{\prime}(S)$, and a vertex $u$ which is not incident to a usable edge with the other end in $S$ is not in $N_{B_{r+1}}^{\prime}(S)$. Suppose that there is no perfect matching in $B_{r+1}$ containing only usable edges. Then by Hall's condition (considering only usable edges), there is a subset $T$ of one of the partite sets in $B_{r+1}$ such that $\left|N_{B_{r+1}}^{\prime}(T)\right|<|T|$. If we choose such a minimal set $S$, then similarly as in the preceding section $S$ satisfies the following conditions:

(i') $\left|N_{B_{r+1}}^{\prime}(S)\right|=|S|-1$,

(ii') $|S| \leq\lceil n / 2\rceil$, and

\footnotetext{
${ }^{2}$ We remark, that our proof method works for slightly smaller values of $c$ as well. However, we do not believe that our method yields an optimal bound on $c$, and therefore we simply require that $c \geq 2$ to keep calculations simple.
} 
(iii') each vertex in $N_{B_{r+1}}^{\prime}(S)$ is adjacent to at least two vertices in $S$ via usable edges.

For $s=1,2, \ldots$, let $Z_{s}$ be a random variable counting the number of minimal sets $S \subseteq V_{2}^{(r+1)}$ in $B_{r+1}$ of size $s$ such that $\left|N_{B_{r+1}}^{\prime}(S)\right|<|S|$, and let $Y_{s}$ be a random variable counting the number of minimal sets $S \subseteq V_{1}^{(r+1)}$ in $B_{r+1}$ of size $s$ such that $\left|N_{B_{r+1}}^{\prime}(S)\right|<|S|$. We set

$$
Z=\sum_{s=1}^{\lceil n / 2\rceil} Z_{s} \text { and } Y=\sum_{s=1}^{\lceil n / 2\rceil} Y_{s}
$$

and first consider the random variable $Z$.

Let us begin with $Z_{1}$. Since each vertex of $V_{2}^{(r+1)}$ has at least $n-r$ potential neighbors in $V_{1}^{(r+1)}$ via usable edges, we have that

$$
\mathbb{E}\left[Z_{1}\right]=n \frac{\left(\begin{array}{c}
n-1 \\
c \log n
\end{array}\right)^{n-r}}{\left(\begin{array}{c}
n \\
c \log n
\end{array}\right)^{n-r}} \leq n \exp \left(-\frac{n-r}{n} c \log n\right)=\Theta\left(n^{1-c}\right),
$$

since $r<m=o(\sqrt{n})$.

Consider now the case when $2 \leq s \leq r+1$. There are $\left(\begin{array}{l}n \\ s\end{array}\right)$ ways of choosing the set $S$, at most $\left(\begin{array}{c}n \\ s-1\end{array}\right)$ ways of choosing the set $N_{B_{r+1}}^{\prime}(S)$, and at most $\left(\begin{array}{l}s \\ 2\end{array}\right)^{s-1}$ ways of realizing (iii'). Moreover, since each vertex in $S$ is matched to $r$ vertices under $M_{1} \cup \cdots \cup M_{r}$, at least $n-r s-s+1$ vertices in $V_{1}^{(r+1)} \backslash N_{B_{r+1}}^{\prime}(S)$ are not adjacent to any vertex in $S$ in $B_{r+1}$, and we thus conclude

$$
\begin{aligned}
\sum_{s=2}^{r+1} \mathbb{E}\left[Z_{s}\right] & \leq \frac{\left(\begin{array}{c}
n \\
s
\end{array}\right)\left(\begin{array}{c}
n \\
s-1
\end{array}\right)\left(\begin{array}{c}
s \\
2
\end{array}\right)^{s-1}\left(\begin{array}{c}
n-2 \\
c \log n-2
\end{array}\right)^{s-1}\left(\begin{array}{c}
n-s \\
c \log n
\end{array}\right)^{n-r s-s+1}}{\left(\begin{array}{c}
n \\
c \log n
\end{array}\right)^{n-r s}} \\
& =O(1) \sum_{s=2}^{r+1} \frac{n^{2 s-1} e^{2 s}(c \log n)^{2 s}}{n^{2(s-1)}} \exp \left(-\frac{s}{n}(n-r s-s+1) c \log n\right) \\
& =O(n) \sum_{s \geq 2}\left(\frac{(c e)^{2} \log ^{2} n}{n^{c\left(1-\lambda_{n}\right)}}\right)^{s},
\end{aligned}
$$

since $r<m=o(\sqrt{n})$, and where $\lambda_{n}$ is some function such that $\lambda_{n} \rightarrow 0$ as $n \rightarrow \infty$. Thus

$$
\sum_{s=2}^{r+1} \mathbb{E}\left[Z_{s}\right]=O\left(n^{-1 / 2}\right)
$$

if $c \geq 2$.

We now deal with the case when $s \geq r+2$. Similarly as above, there are $\left(\begin{array}{l}n \\ s\end{array}\right)$ ways of choosing the set $S$, at most $\left(\begin{array}{c}n \\ s-1\end{array}\right)$ ways of choosing the set $N_{B_{r+1}}^{\prime}(S)$, and at most $\left(\begin{array}{l}s \\ 2\end{array}\right)^{s-1}$ ways of realizing (iii'). Note further that if a vertex $u \in V_{1}^{(r+1)} \backslash N_{B_{r+1}}^{\prime}(S)$ is adjacent to some vertex $v \in S$, then $u v \in M_{1} \cup \cdots \cup M_{r}$, and thus there are at most $\left(\begin{array}{c}n-s+r \\ c \log n\end{array}\right)$ ways of choosing the neighbors of a vertex 
in $V_{1}^{(r+1)} \backslash N_{B_{r+1}}^{\prime}(S)$. Hence,

$$
\begin{aligned}
\sum_{s=r+2}^{\lceil n / 2\rceil} \mathbb{E}\left[Z_{s}\right] & \leq \sum_{s=r+2}^{\lceil n / 2\rceil} \frac{\left(\begin{array}{c}
n \\
s
\end{array}\right)\left(\begin{array}{c}
n \\
s-1
\end{array}\right)\left(\begin{array}{c}
s \\
2
\end{array}\right)^{s-1}\left(\begin{array}{c}
n-2 \\
c \log n-2
\end{array}\right)^{s-1}\left(\begin{array}{c}
n-s+r \\
c \log n
\end{array}\right)^{n-s+1}}{\left(\begin{array}{c}
n \\
c \log n
\end{array}\right)^{n}} \\
& =O(n) \sum_{s=r+2}^{\lceil n / 2\rceil} e^{2 s}(c \log n)^{2 s} \exp \left(-\frac{s-r}{n}(n-s+1) c \log n\right) \\
& =O\left(n^{1+\frac{r c}{2}}\right) \sum_{s \geq r+2}\left(\frac{(c e)^{2} \log ^{2} n}{n^{c / 2}}\right)^{s}=O\left(n^{-1 / 2}\right),
\end{aligned}
$$

if $c \geq 2$. It follows from this and (6)-(7) that $\mathbb{P}[Z>0]=O\left(n^{-1 / 2}\right)$ if $c \geq 2$.

Let us now verify that whp there is no set $S \subseteq V_{1}^{(r+1)}$ in $B_{r+1}$ such that $\left|N_{B_{r+1}}^{\prime}(S)\right|<|S|$. Using the same estimates as above and noting that since each vertex of $V_{1}^{(r+1)}$ is matched to $r$ vertices under $M_{1} \cup \cdots \cup M_{r}$, there are at most $\left(\begin{array}{c}s-1+r \\ c \log n\end{array}\right)^{s}$ ways of choosing the neighbors for a vertex in $S$ in $B_{r+1}$, we conclude that

$$
\begin{aligned}
\mathbb{P}[Y>0] & \leq \sum_{s=\max \{c \log n-r+1,1\}}^{\lceil n / 2\rceil} \mathbb{E}\left[Y_{s}\right] \\
& \leq \sum_{s=\max \{c \log n-r+1,1\}}^{\lceil n / 2\rceil} \frac{\left(\begin{array}{c}
n \\
s
\end{array}\right)\left(\begin{array}{c}
n \\
s-1
\end{array}\right)\left(\begin{array}{c}
s-1+r \\
c \log n
\end{array}\right)^{s}\left(\begin{array}{c}
n \\
c \log n
\end{array}\right)^{n-s}}{\left(\begin{array}{c}
n \\
c \log n
\end{array}\right)^{n}} \\
& =O(1) \sum_{s=2}^{\lceil n / 2\rceil}\left(\frac{e n}{s}\right)^{s}\left(\frac{e n}{s-1}\right)^{s-1}\left(\frac{s-1+r}{n}\right)^{s c \log n} .
\end{aligned}
$$

The last sum can be handled similarly as the sum in (4): split the sum in (8) into two parts $\Delta_{1}$ and $\Delta_{2}$, so that $\Delta_{1}$ is a sum from 2 to $n / w_{n}-r+1$ (where $w_{n} \rightarrow \infty$ arbitrarily slowly as $n \rightarrow \infty$ ) and $\Delta_{2}$ contains the rest of the terms. Proceeding along the same lines as in the last part of the proof of Theorem 1.3, it is then straightforward to verify that $\mathbb{P}[Y>0]=O\left(n^{-1 / 2}\right)$ if $c \geq 2$. This completes the proof of Theorem 1.5.

Remark 3.1. It should be noted that if $m=\Theta(1)$, then it is suffices to require that $c>1$ in the proof of Theorem 1.5. This fact together with Theorem 1.3 shows that for $K_{m, n}$ the property of being colorable from a random $(f(n),\{1, \ldots, n\}$ )-list assignment has a sharp threshold at $f(n)=\log n$ if $m=\Theta(1)$.

\section{References}

[1] L. J. Andrén, C. J. Casselgren, L.-D. Öhman, Avoiding arrays of odd order by Latin squares, Combinatorics, Probability 85 Computing 22 (2013), 184-212.

[2] C. J. Casselgren, Vertex coloring complete multipartite graphs from random lists of size 2, Discrete Mathematics 311 (2011), 1150-1157.

[3] C. J. Casselgren, Coloring graphs from random lists of size 2, European Journal of Combinatorics 33 (2012), 168-181. 
[4] C. J. Casselgren, Coloring graphs from random lists of fixed size Random Structures \& Algorithms 44 (2014), 317-327.

[5] C. J. Casselgren On some graph coloring problems, Doctoral thesis, Umeå University, Umeå, 2011.

[6] P. Erdős, A. L. Rubin, H. Taylor, Choosability in graphs, Proceedings West Coast Conf. on Combinatorics, Graph Theory and Computing, Congressus Numerantium XXVI (1979), 125157.

[7] F. Galvin, The list chromatic index of a bipartite multigraph, Journal of Combinatorial Theory Series B 63 (1995), 153-158.

[8] S. Janson, T. Łuczak, A. Ruciński, Random Graphs, Wiley, New York, 2000.

[9] M. Krivelevich, A. Nachmias, Coloring powers of cycles from random lists, European Journal of Combinatorics 25 (2004), 961-968.

[10] M. Krivelevich, A. Nachmias, Coloring Complete Bipartite Graphs from Random Lists, Random Structures and Algorithms 29 (2006), 436-449.

[11] V. G. Vizing, Coloring the vertices of a graph with prescribed colors, Metody Diskretnogo Analiza Teorii Kodov i Skhem 29 (1976), 3-10 (in Russian). 